The Astrophysical Journal Supplement Series, 154:222-228, 2004 September

(C) 2004. The American Astronomical Society. All rights reserved. Printed in U.S.A.

\title{
INFRARED ARRAY CAMERA (IRAC) OBSERVATIONS OF M81
}

\author{
S. P. Willner, ${ }^{1}$ M. L. N. Ashby, ${ }^{1}$ P. Barmby, ${ }^{1}$ G. G. Fazio, ${ }^{1}$ M. Pahre, ${ }^{1}$ H. A. Smith, ${ }^{1}$ Robert C. Kennicutt, $\mathrm{Jr}^{2},{ }^{2}$ Daniela Calzetti, ${ }^{3}$ \\ Daniel A. Dale, ${ }^{4}$ B. T. Draine, ${ }^{5}$ Michael W. Regan, ${ }^{3}$ S. Malhotra, ${ }^{3}$ Michele D. Thornley, ${ }^{3,6}$ P. N. Appleton, ${ }^{7}$ \\ D. Frayer, ${ }^{7}$ G. Helou, ${ }^{7}$ S. Stolovy, ${ }^{7}$ and L. Storrie-Lombardi ${ }^{7}$ \\ Received 2004 March 26; accepted 2004 May 27
}

\begin{abstract}
Infrared Array Camera (IRAC) images of M81 show three distinct morphological constituents: a smooth distribution of evolved stars with bulge, disk, and spiral arm components; a clumpy distribution of dust emission tracing the spiral arms; and a pointlike nuclear source. The bulge stellar colors are consistent with M-type giants, and the disk colors are consistent with a slightly younger population. The dust emission generally follows the blue and ultraviolet emission, but there are large areas that have dust emission without ultraviolet and smaller areas with ultraviolet but little dust emission. The former are presumably caused by extinction, and the latter may be due to cavities in the gas and dust created by supernova explosions. The nucleus appears fainter at $8 \mu \mathrm{m}$ than expected from ground-based $10 \mu \mathrm{m}$ observations made 4 years ago.
\end{abstract}

Subject headings: dust, extinction — galaxies: individual (M81) — galaxies: ISM — galaxies: spiral — galaxies: stellar content — infrared: galaxies

\section{INTRODUCTION}

Spiral galaxies are valuable laboratories for stellar evolution, stellar population, and star formation studies. Detailed observations of the nearest galaxies are especially valuable, because they allow us to characterize the integrated properties of the stellar and interstellar components and their global relationships, while maintaining sufficient spatial resolution and sensitivity to study the properties of individual regions.

Infrared studies of spiral galaxies yield insight into both the evolved stellar content and star formation. Because red giant stars have their peak emission in the near-infrared, this wavelength range is most directly related to the underlying stellar mass (e.g., Kauffmann \& Charlot 1998; Bell \& de Jong 2001). Emission from the hottest dust grains in star-forming regions is detectable at wavelengths longer than about $3 \mu \mathrm{m}$, but grain emission only becomes a significant fraction of the overall emission at $\lambda \gtrsim 5 \mu \mathrm{m}$ with the rise of the "aromatic features" (Helou et al. 2000; Lu et al. 2003). These features are usually attributed to vibrational modes of benzene-ringbased materials often called polycyclic aromatic hydrocarbons (PAHs; Leger \& Puget 1984). This emission is dominated by transient heating from single-photon events, so there is no temperature information in the emission colors.

Spitzer Space Telescope observations of spiral galaxies offer a tremendous advance over previous capabilities. The vast improvement in sensitivity gives the ability to map entire galaxies quickly, and the wavelength coverage allows direct tracing of the stellar and nonstellar components (e.g., Pahre

\footnotetext{
${ }^{1}$ Harvard-Smithsonian Center for Astrophysics, 60 Garden Street, Cambridge, MA 02138.

2 Steward Observatory, University of Arizona, Tucson, AZ 85721.

3 Space Telescope Science Institute, 3700 San Martin Drive, Baltimore, MD 21218

4 Department of Physics and Astronomy, University of Wyoming, Laramie, WY 82071.

5 Princeton University Observatory, Princeton, NJ 08544.

6 Department of Physics, Bucknell University, Lewisburg, PA 17837.

7 Spitzer Science Center, California Institute of Technology, 1200 East California Boulevard, Pasadena, CA 91125.
}

et al. 2004, who discuss classification schemes based on these components). Spitzer also provides sufficient angular resolution to map individual clouds and star-forming regions in nearby galaxies; the Infrared Array Camera resolution of $\leq 2^{\prime \prime}$ corresponds to a linear scale of $\leq 40 \mathrm{pc}$ at a distance of $4 \mathrm{Mpc}$.

This paper reports initial observations of M81 with the Infrared Array Camera (IRAC; Fazio et al. 2004) on Spitzer. Gordon et al. (2004) report companion observations at longer wavelengths, and Helou et al. (2004) report similar observations of NGC 300. M81 is one of the nearest large spiral galaxies at a distance of 3.63 Mpc (Karachentsev et al. 2002). Its Hubble type is Sab (de Vaucouleurs et al. 1991), and its farinfrared luminosity is $L_{\mathrm{FIR}}=3 \times 10^{9} L_{\odot}$ (Rice et al. 1988). M81 is a member of a group with $\sim 25$ companions and has recently interacted with M82 and NGC 3077 (Yun et al. 2000) and perhaps NGC 2976 (Appleton \& van der Hulst 1988). With isophotal major and minor axis diameters of $27^{\prime} \times 14^{\prime}$, M81 is an excellent target for initial Spitzer observations. M81 also is the largest galaxy in the Spitzer Infrared Nearby Galaxies Survey (SINGS; Kennicutt et al. 2003), and these early observations provide a demonstration of the scientific potential of SINGS for its full 75 galaxy sample.

\section{OBSERVATIONS}

IRAC observations of M81 were made on 2003 November 6. Each of 23 positions was observed with six 12 s frames: two repeats at each of three dither positions. Positions near the center were observed last to minimize the effects of residual images. The visible disk of the galaxy was observed by both of IRAC's side-by-side fields of view, but the extended sky coverage is toward the northeast for 3.6 and $5.8 \mu \mathrm{m}$ and toward the southwest for 4.5 and $8.0 \mu \mathrm{m}$.

The data were processed with version 8.5.0 of the Spitzer Science Center (SSC) online pipeline, using calibration files ("skyflats" and "skydarks") derived from in-flight data. Further processing was carried out in IRAF: "skydarks" constructed by median-combining data well outside the galaxy were subtracted from 3.6 and $5.8 \mu \mathrm{m}$ frames, a similarly constructed "skyflat" was divided into the $8.0 \mu \mathrm{m}$ frames, and background levels of 
frames in all four bands were set to approximately zero by subtracting the median sky levels. The SSC "background matching" software was used to refine this procedure. Image artifacts $^{8}$ were corrected (for cosmetic purposes) with the IRAF IMEDIT task. Mosaics combining all the data were made with the 2004 January 17 version of the SSC software MOPEX. Mosaics for all four bands were made on the same sky grid using 0 ."86 pixels. Because cosmic rays have different appearances in the four bands, different MOPEX settings were used to optimize cosmic-ray rejection. Figure 1 shows the four resulting images. ${ }^{9}$

\section{ANALYSIS}

Inspection of Figure 1 (Plate 1) shows three distinct morphological components: a smooth distribution of starlight prominent in the 3.6 and $4.5 \mu \mathrm{m}$ images, a clumpy distribution of dust emission seen at 5.8 and $8 \mu \mathrm{m}$, and a pointlike source at the nucleus seen at $4.5 \mu \mathrm{m}$ and longer wavelengths. These components are discussed in turn below.

Quantitative analysis was based on fitting elliptical isophotes (Pahre et al. 2004) to all four images starting at a radius of $5^{\prime \prime}$. The isophotes as a function of semimajor axis were then fitted to disk+bulge models. Similar models were also fitted to circular aperture magnitudes, and the differences are taken to indicate the uncertainties. Observed quantities have no extinction correction; Galactic extinction is negligible (Schlegel et al. 1998), and extinction internal to M81 is patchy and uncertain. $^{10}$

\subsection{Stellar Content}

At 3.6 and $4.5 \mu \mathrm{m}$, the isophotes are well fitted by the disk+bulge models as shown in Figure 2. Parameters are given in Table 1. The fitting also determines total magnitudes, which are 3.52 and 3.61 (relative to Vega $=0$ ) at isophotal wavelengths of 3.54 and $4.50 \mu \mathrm{m}$ (Fazio et al. 2004). Uncertainties are limited by the Spitzer calibration uncertainty of $10 \%$. Comparison with the 2MASS $K_{S}$ total magnitude 3.83 (Jarrett et al. 2003) shows that any nonstellar contribution at $3.6 \mu \mathrm{m}$ is small. The derived color $K_{S}-[3.6]=0.31$ is the same within the uncertainties as $K-L=0.25 \pm 0.05$ for normal galaxies (Willner et al. 1984). Likewise, [3.6] $-[4.5]=-0.09$ is very close to the expected -0.15 for an M0 III star (M. Cohen 2004, private communication), a spectral type representative of old stellar populations (Frogel 1985). ${ }^{11}$

Despite the overall good fit of the [3.6] - [4.5] color to late type stellar colors over most of the galaxy, there are regions that show redder colors. One region is the nucleus, discussed below. In addition, the bulge and disk show different overall colors: $[3.6]-[4.5]=-0.17$ for the bulge and -0.04 for the disk. All explanations for this color difference point to a disk generally younger than the bulge. A stellar population even slightly younger - dominated by $\mathrm{K}$ stars instead of $\mathrm{M}$ starswould have [3.6] - [4.5] close to zero. A greater proportion of dusty interstellar matter in the disk may also cause reddening

\footnotetext{
8 Artifacts include banding, muxbleed, and column pulldown and are described in the IRAC Data Handbook at http://ssc.spitzer.caltech.edu/irac/dh/.

9 Data files are available via http://cfa-www.harvard/edu/irac/publications.

${ }^{10}$ For example, Kaufman et al. (1989a) find $A_{V}>8$ for some of the narrow dust lanes, but extinction is much smaller over most of the disk. $A_{V}=8$ corresponds to $E([3.6]-[4.5]) \approx 0.09$, which is near the limit of detection in the current data.

${ }^{11}$ The negative, i.e., blue, color for M stars is because of $\mathrm{CO}$ absorption in $\mathrm{M}$ star atmospheres in the $4.5 \mu \mathrm{m}$ band. $\mathrm{K}$ stars have weaker CO absorption and are therefore redder than $\mathrm{M}$ stars in the [3.6] - [4.5] color.
}

or, if the dust is heated, show up in emission, but dust is likely to be a small contributor to the color difference. ${ }^{12}$ There is also a small color gradient within the bulge. In contrast to the disk fit, which shows the same effective radius at 3.6 and $4.5 \mu \mathrm{m}$, the bulge fit shows a larger effective radius at 3.6 than at $4.5 \mu \mathrm{m}$. This means the inner regions of the bulge are redder than the outer regions and may indicate a younger stellar component near the nucleus.

The arm/interarm surface brightness ratio is about $0.5 \mathrm{mag}$ at $3.6 \mu \mathrm{m}$, comparable to values seen in other galaxies at $2.2 \mu$ m (e.g., Rix \& Rieke 1993; Rix \& Zaritsky 1995; Grauer $\&$ Rieke 1998). This ratio should be a good measure of the mass density enhancement in the arms. ${ }^{13}$ Nearly the same arm/interarm ratio is found from a blue image (Cheng et al. 1997) when the exact same areas of the galaxy are compared. This agrees with ratios found by Elmegreen et al. (1989). In fact, the $3.6 \mu \mathrm{m}$ contrast is smaller inside a $5^{\prime}$ radius and larger outside, just like the contrast in blue light.

However, the qualitative appearance in blue light differs from that at $3.6 \mu \mathrm{m}$; the latter shows a smoother distribution, being less affected by star clusters or extinction patches.

\subsection{Dust Content}

One of the most striking results from the IRAC images is the complex structure of the nonstellar emission, as traced in the 5.8 and $8 \mu \mathrm{m}$ images. Based on the morphology and colors, we assume that the $3.6 \mu \mathrm{m}$ image is a direct measure of the stellar light. We have therefore subtracted the $3.6 \mu \mathrm{m}$ image, scaled according to the expected color of an M0 star (Pahre et al. 2004), from the 5.8 and $8 \mu \mathrm{m}$ images to produce images of nonstellar emission. The $8 \mu \mathrm{m}$ nonstellar image is shown in Figure 3 (Plate 2). Nonstellar flux densities are 2.3 and $5.9 \mathrm{Jy}$ at 5.8 and $8.0 \mu \mathrm{m}$, respectively, $35 \%$ and $69 \%$ of the emission at these wavelengths.

Qualitatively, the nonstellar emission traces the same spiral structure as other gas tracers such as the H I (cf. Rots \& Shane 1975; Appleton et al. 1981; Adler \& Westpfahl 1996) and optical dust lanes, as well as young stellar tracers including $\mathrm{H} \alpha$, ultraviolet continuum emission (Marcum et al. 2001), and the radio continuum (Kaufman et al. 1989b). When the structure of the IR emission is examined in more detail, however, it is most strongly correlated with the optical dust lanes (as might be expected). Figure 3 shows large regions where there is strong emission at $8 \mu \mathrm{m}$ but no detectable ultraviolet emission. Detectable dust emission extends to the outer reaches of the star-forming disk but not as far as the outer H I spiral arms (Adler \& Westpfahl 1996), showing a decline in the product of PAH abundance and intensity of the stellar radiation.

The nonstellar emission is both more spatially extended than the stellar distribution (as shown in Fig. $2 d$ by the rising ratio of nonstellar to stellar emission beyond $100^{\prime \prime}$ ) and much

\footnotetext{
12 If the color difference between disk and bulge is entirely due to emission from transient-heated hot dust grains in the disk, and the dust emission follows the $f_{\nu} \propto \nu^{+0.65}$ spectrum found by Helou et al. (2000), the dust contribution to the $3.6 \mu \mathrm{m}$ emission would be $7 \%$ of the disk flux and $4 \%$ of the total flux. The Li \& Draine (2001) dust model predicts a steeper slope, which would make the $3.6 \mu \mathrm{m}$ dust contribution $13 \%$ of the disk emission at that wavelength.

${ }^{13}$ If short-lived red supergiants contribute significantly to the light in the spiral arms but little to the interarm regions, the arm/interarm mass ratio would be smaller than indicated by the relative surface brightnesses. However, Rix \& Rieke (1993) found for M51 that red supergiants are significant only in a single small patch and not in the overall emission.
} 


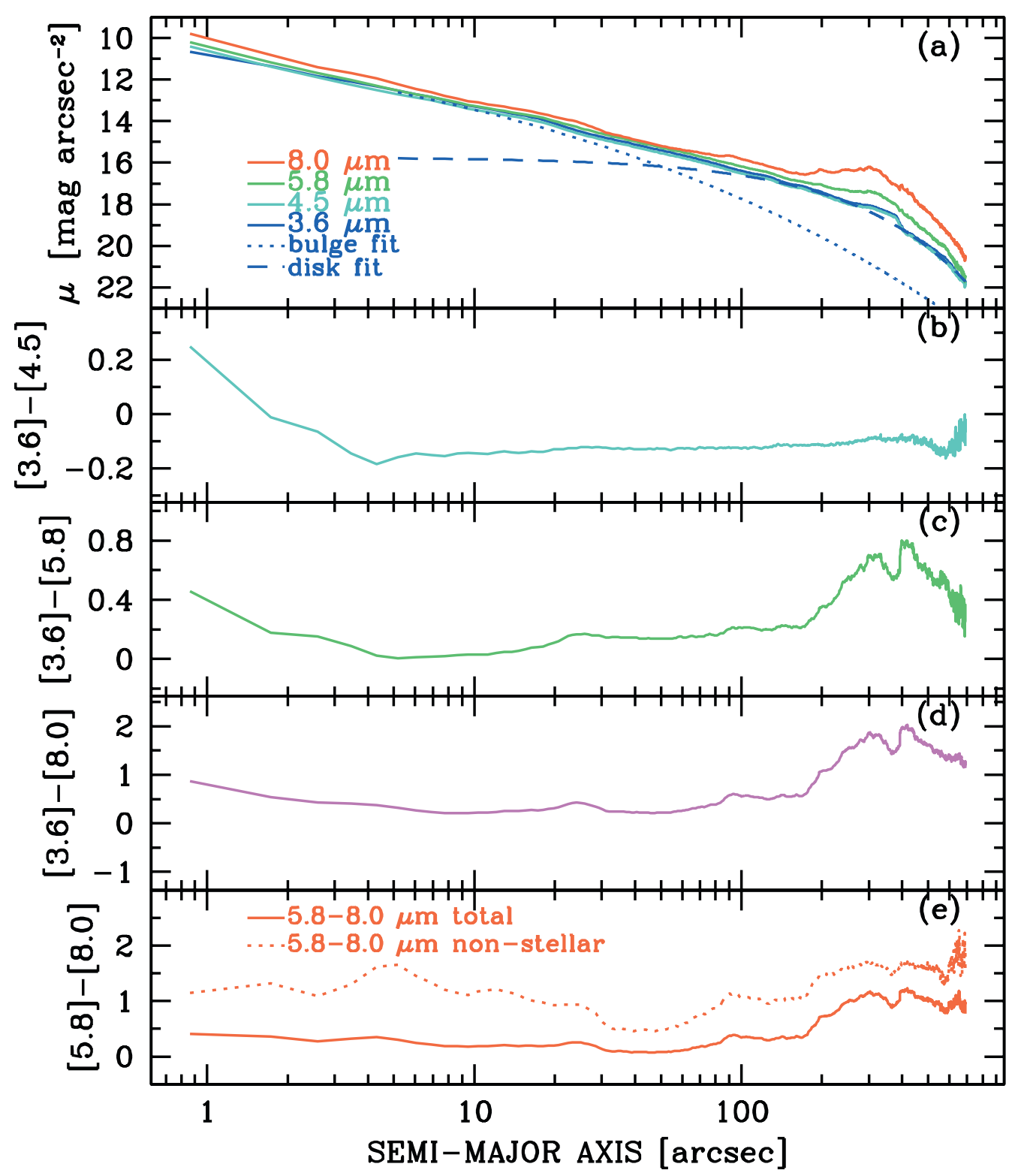

FIG. 2.- Surface brightness profiles in elliptical isophotes. Solid lines in $(a)$ show the measured surface brightness profiles for all four IRAC bands. Dashed lines show the bulge +disk model for $3.6 \mu \mathrm{m}$. Panels $(b),(c)$, and $(d)$ and the solid line in $(e)$ show the measured surface brightness profiles for three different IRAC colors. The dashed line in $(e)$ shows the surface brightness profile for the nonstellar emission alone (i.e., after starlight has been subtracted) in the [5.8] $-[8.0]$ color.

TABLE 1

M81 Stellar Properties

\begin{tabular}{|c|c|c|}
\hline Parameter & $3.6 \mu \mathrm{m}^{\mathrm{a}}$ & $B^{\mathrm{b}}$ \\
\hline Bulge total magnitude........... & $4.62 \pm 0.05$ & 8.99 \\
\hline Bulge half-light semimajor axis $a_{B}(\operatorname{arcsec}) \ldots \ldots \ldots \ldots \ldots \ldots$ & 54 & 85 \\
\hline 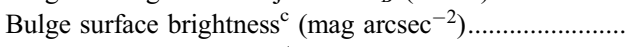 & $14.86 \pm 0.03$ & 20.29 \\
\hline 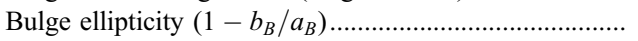 & 0.30 & 0.26 \\
\hline 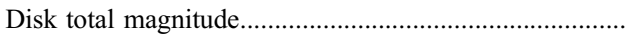 & $4.01 \pm 0.05$ & 8.23 \\
\hline Disk half-light semimajor axis $a_{D}(\operatorname{arcsec}) \ldots \ldots \ldots \ldots \ldots \ldots \ldots \ldots$ & 242 & 288 \\
\hline 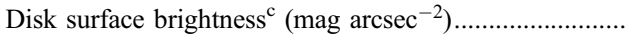 & $17.09 \pm 0.23$ & 21.71 \\
\hline 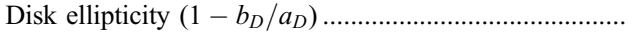 & 0.53 & 0.53 \\
\hline 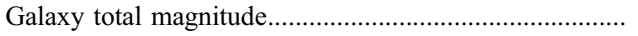 & $3.52 \pm 0.02$ & 7.79 \\
\hline Bulge-to-disk ratio & $0.57 \pm 0.05$ & 0.49 \\
\hline
\end{tabular}

${ }^{a}$ Indicated uncertainties are those from the fitting procedure; they do not include overall calibration uncertainties of approximately $10 \%$.

b Parameters from Möllenhoff (2004). Bulge fit at $B$ is based on an $r^{-0.29}$ profile instead of $r^{-0.25}$ as used for $3.6 \mu \mathrm{m}$.

${ }^{\mathrm{c}}$ Mean surface brightness for area within half-light ellipse. 
clumpier (as shown in Fig. 1). The arm/interarm ratio is 3 in the $8 \mu \mathrm{m}$ image (including starlight), similar to the ratio for NGC 6946 at 7 and $15 \mu \mathrm{m}$ (Malhotra et al. 1996). The nonstellar image (i.e., with starlight subtracted) is so clumpy that an "arm surface brightness" is virtually meaningless, but even modest clumps have surface brightnesses an order of magnitude greater than interarm regions, ${ }^{14}$ and the brightest clumps are more than 3 times brighter still.

The interstellar medium emission at the 5.8 and $8 \mu \mathrm{m}$ is likely dominated by aromatic features usually attributed to $\mathrm{PAH}$, as demonstrated for instance by the ISO PHT spectra of galaxies analyzed by $\mathrm{Lu}$ et al. (2003). Rigopoulou et al. (1999) observed the central region of M81 $\left(24^{\prime \prime} \times 24^{\prime \prime}\right.$ beam) with ISOPHOT-S and detected emission in the $7.7 \mu \mathrm{m} \mathrm{PAH}$ feature but found a small feature/continuum ratio of less than 0.115 . The low ratio compared to other galaxies may result from the large angular size of M81 relative to the more distant galaxies observed and thus the lesser disk contribution in the same fixed beam size. Both starlight from the bulge and active galactic nucleus (AGN) emission ( $\S 3.3$ ) in the M81 nucleus will dilute the aromatic feature emission, which mostly comes from the disk (Fig. 3). Aromatic feature emission measured with ISOCAM in the $6.75 \mu \mathrm{m}$ filter correlates well with $\mathrm{H} \alpha$ emission (Roussel et al. 2001) (although the $\mathrm{H} \alpha$ to $15 \mu \mathrm{m}$ correlation may be stronger-Sauvage et al. 1996). PAH at $6.75 \mu \mathrm{m}$ was therefore proposed as a reliable star formation estimator for typical galaxy disks. However, old, cool stars can also excite aromatic feature emission (Li \& Draine 2002), and such emission is therefore not expected to be a perfect star formation indicator, any more than far-infrared luminosity or any other indicator.

Figure 3 shows that the nonstellar dust emission overall tracks the near-ultraviolet emission (NUV), but there are both regions with dust emission and no NUV and with NUV but no dust emission. PAH without NUV can be explained by dust extinction; 1 mag of visual extinction, which would heavily attenuate the NUV, corresponds to an undetectable $0.01 \mathrm{mag}$ of differential extinction between 3.6 and $4.5 \mu \mathrm{m}$. Seeing NUV light but not dust emission is harder to explain, but perhaps cavities cleared by supernova explosions are responsible. A signature of such cavities is clumpy $\mathrm{H} \alpha$ (Cheng et al. 1997) and $8 \mu \mathrm{m}$ emission surrounding an area dark at these wavelengths but bright in NUV. This morphology can be explained by a supernova explosion having cleared gas and dust from a young star cluster, leaving behind and revealing the hot stars.

\subsection{Nucleus}

The nucleus of M81 has been classified as a LINER (Heckman 1980) or Seyfert 1 (Peimbert \& Torres-Peimbert 1981) with an estimated black hole mass $7 \times 10^{7} M_{\odot}$ (Devereux et al. 2003). Like many galactic nuclei with inferred black holes, the M81 nucleus seems relatively inactive. Nuclear emission varies by a factor of about 2 in visible light (Devereux et al. 2003) and near $10 \mu \mathrm{m}$ (Grossan et al. 2001) on timescales estimated at about 25 years. The ground-based $10 \mu \mathrm{m}$ data at $\sim$ arcsecond resolution suggest a pointlike nucleus.

The new IRAC data suggest that the nuclear source has varied in the previous 4 years. IRAC flux densities in a

\footnotetext{
14 The interarm regions probably have nonzero surface brightness, but the uncertainties are large and hard to quantify because the result depends so critically on what stellar spectrum is subtracted and on the still poorly known IRAC calibration for extended sources.
}

3."5 square aperture (including starlight) are 109, 75, 65, and $56 \mathrm{mJy}$ at 3.6 to $8 \mu \mathrm{m}$, respectively, with uncertainties of about $10 \%{ }^{15}$ The $8 \mu \mathrm{m}$ flux density can be compared with the $199910 \mu \mathrm{m}$ flux density of $159 \mathrm{mJy}$ (Grossan et al. 2001). The spectral shape of the nuclear emission between 8 and $10 \mu \mathrm{m}$ is not known, although the ISO spectrum in a $24^{\prime \prime}$ beam shows a steep rise (Rigopoulou et al. 1999). Nevertheless, it seems hard to account for the difference in measured flux densities (159 mJy at $10 \mu \mathrm{m}$ in 1999; $56 \mathrm{mJy}$ at $8 \mu \mathrm{m}$ in 2003) by spectral shape alone. If the difference is due to variability, it will strongly support the idea that we are seeing an AGN component.

In order to examine the nuclear morphology, the bulge+disk model fits were subtracted from the IRAC images at each wavelength. The results are shown in Figure 3. A residual pointlike source is quite evident at $4.5 \mu \mathrm{m}$ and longer wavelengths and might be present at $3.6 \mu \mathrm{m}$ as well, although the brightness of the stellar bulge at $3.6 \mu \mathrm{m}$ and nearby negative values in the difference image make it hard to be sure. Flux densities of the apparent pointlike source at the four IRAC wavelengths are $6,21,22$, and $39 \mathrm{mJy}$, respectively, with the first being uncertain by more than a factor of 2 . Davidge $\&$ Courteau (1999) found an unresolved source at the M81 nucleus on the basis of $J-K$ color, and Quillen et al. (2001) measured a flux density for the point source of $13 \mathrm{mJy}$ at $1.6 \mu \mathrm{m}$. Considering the uncertainties and possible variability, the spectral energy distribution from 1.6 to $8 \mu \mathrm{m}$ might be consistent with either a power law $\left(F_{\nu} \propto \nu^{-0.7}\right)$ or emission from dust having a temperature range of $400-1000 \mathrm{~K}$ and emissivity that decreases with wavelength.

The images also show a filament or bar of material leading from a ringlike structure at $\sim 1 \mathrm{kpc}\left(1^{\prime}\right)$ to an inner disk and then to the AGN. The prominence of the ring at $8 \mu \mathrm{m}$ suggests that much of its emission is from dust and probably mostly aromatic features. These inner features are probably related to the structures seen in HST H $\alpha$ images (Devereux et al. 2003).

\section{CONCLUSIONS}

1. IRAC images of M81 delineate three components by both morphology and energy distribution: evolved stars organized in bulge and disk components; a clumpy, dusty interstellar medium with star-forming regions; and a pointlike active nucleus.

2. The disk is redder than the bulge in the $[3.6]-[4.5]$ color. This could be explained by reddening in the disk, by small amounts of dust emission in the disk, or by a younger stellar population in the disk.

3. The arm/interarm surface brightness contrast at $3.6 \mu \mathrm{m}$ is $0.5 \mathrm{mag}$, in agreement with previous results in blue light. For normal stellar populations, this value should represent the stellar mass density contrast.

4. The nucleus of M81 shows an apparent point source. Its flux density appears to have decreased by something like a factor of 3 in the past 4 years, and its SED can be represented by a nonthermal power law or by hot dust emission.

5. Star-forming regions are identified by their dust emission, probably mostly in the aromatic feature bands. Overall the dust

\footnotetext{
15 These flux densities are relative to Vega flux densities of $277.5,179.5$, 116.6, and 63.1 Jy and are without subtracting any stellar component. They include corrections for IRAC aperture effects and are thus directly comparable to ground-based observations made with a large chopper throw. Chopping with a small throw (e.g., 9"6 as used by Grossan et al.) would give smaller flux densities than with a large throw.
} 
emission traces the spiral arms as seen in visible and nearultraviolet light, but there are many regions where dust emission is present and NUV is absent and some where the reverse is true. The ubiquity of dust emission implies that absorption affects both the distribution and amount of NUV light seen from star-forming regions.

This work is based on observations made with the Spitzer Space Telescope, which is operated by the Jet Propulsion Laboratory, California Institute of Technology, under NASA contract 1407. Support for this work was provided by NASA through contract 1256790 issued by JPL/Caltech. Support for the IRAC instrument was provided by NASA through contract 960541 issued by JPL. M. A. P. acknowledges NASA/LTSA grant NAG5-10777.

IRAF is distributed by the National Optical Astronomy Observatories, which are operated by the Association of Universities for Research in Astronomy, Inc., under cooperative agreement with the National Science Foundation.

This research has made use of the NASA/IPAC Extragalactic Database (NED), which is operated by the Jet Propulsion Laboratory, California Institute of Technology, under contract with the National Aeronautics and Space Administration.

The data set used in this analysis (Spitzer AOR key 0006629120) is available through a link in the electronic edition.
Adler, D. S., \& Westpfahl, D. J. 1996, AJ, 111, 735

Appleton, P. N., Davies, R. D., \& Stephenson, R. J. 1981, MNRAS, 195, 327

Appleton, P. N., \& van der Hulst, J. M. 1988, MNRAS, 234, 957

Bell, E. F., \& de Jong, R. S. 2001, ApJ, 550, 212

Cheng, K.-P., et al. 1997, UV/Visible Sky Gallery, CD-ROM (Washington: NASA/NED)

Davidge, T. J., \& Courteau, S. 1999, AJ, 117, 2781

de Vaucouleurs, G., de Vaucouleurs, A., Corwin, H. G., Buta, R. J., Paturel, G., \& Fouque, P. 1991, Third Reference Catalog of Bright Galaxies (Berlin: Springer)

Devereux, N., Ford, H., Tsvetanov, Z., \& Jacoby, G. 2003, AJ, 125, 1226

Elmegreen, B. G., Seiden, P. E., \& Elmegreen, D. M. 1989, ApJ, 343, 602

Fazio, G. G., et al. 2004, ApJS, 154, 10

Frogel, J. A. 1985, ApJ, 298, 528

Gordon, K. D., et al. 2004, ApJS, 154, 215

Grauer, A. D., \& Rieke, M. J. 1998, ApJS, 116, 29

Grossan, B., Gorjian, V., Werner, M., \& Ressler, M. 2001, ApJ, 563, 687

Heckman, T. M. 1980, A\&A, 87, 152

Helou, G., Lu, N. Y., Werner, M. W., Malhotra, S., \& Silbermann, N. A. 2000, ApJ, 532, L21

Helou, G., et al. 2004, ApJS, 154, 253

Jarrett, T. H., Chester, T., Cutri, R., Schneider, S. E., \& Huchra, J. P. 2003, AJ, 125,525

Karachentsev, I. D., et al. 2002, A\&A, 383, 125

Kauffmann, G., \& Charlot, S. 1998, MNRAS, 297, L23

Kaufman, M., Elmegreen, D. M., \& Bash, F. N. 1989a, ApJ, 345, 697

Kaufman, M., et al. 1989b, ApJ, 345, 674

Kennicutt, R. C., et al. 2003, PASP, 115, 928

\section{REFERENCES}

Leger, A., \& Puget, J.-L. 1984, A\&A, 137, L5

Li, A., \& Draine, B. T. 2001, ApJ, 554, 778 2002, ApJ, 572, 232

Lu, N., et al. 2003, ApJ, 588, 199

Malhotra, S., et al. 1996, A\&A, 315, L161

Marcum, P. M., et al. 2001, ApJS, 132, 129

Möllenhoff, C. 2004, A\&A, 415, 63

Pahre, M. A., Ashby, M. L. N., Fazio, G. G., \& Willner, S. P. 2004, ApJS, 154,235

Peimbert, M., \& Torres-Peimbert, S. 1981, ApJ, 245, 845

Quillen, A. C., McDonald, C., Alonso-Herrero, A., Lee, A., Shaked, S., Rieke, M. J., \& Rieke, G. H. 2001, ApJ, 547, 129

Rice, W., Lonsdale, C. J., Soifer, B. T., Neugebauer, G., Koplan, E. L., Lloyd, L. A., de Jong, T., \& Habing, H. J. 1988, ApJS, 68, 91

Rigopoulou, D., Spoon, H. W. W., Genzel, R., Lutz, D., Moorwood, A. F. M., \& Tran, Q. D. 1999, AJ, 118, 2625

Rix, H., \& Rieke, M. J. 1993, ApJ, 418, 123

Rix, H., \& Zaritsky, D. 1995, ApJ, 447, 82

Rots, A. H., \& Shane, W. W. 1975, A\&A, 45, 25

Roussel, H., Sauvage, M., Vigroux, L., \& Bosma, A. 2001, A\&A, 372, 427

Sauvage, M., et al. 1996, A\&A, 315, L89

Schlegel, D. J., Finkbeiner, D. P., \& Davis, M. 1998, ApJ, 500, 525

Willner, S. P., Fabbiano, G., Elvis, M., Ward, M., Longmore, A., \& Lawrence, A. 1984, PASP, 96, 143

Yun, M. S., Ho, P. T. P., \& Lo, K. Y. 2000, in ASP Conf. Ser. 217, Imaging at Radio through Submillimeter Wavelengths, ed. J. G. Mangum \& S. J. E. Radford (San Francisco: ASP), 374 


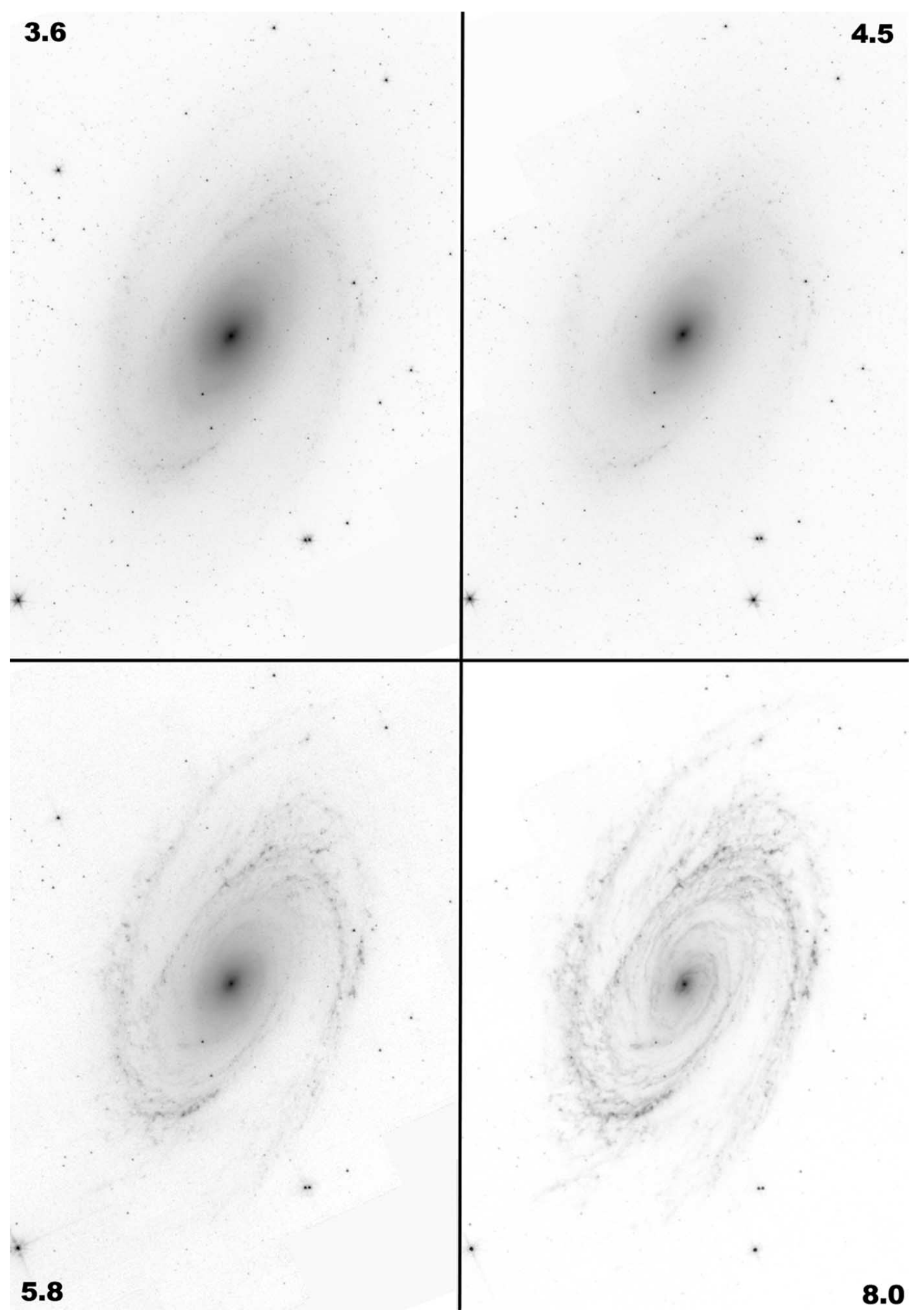

FIG. 1.-IRAC images in the four bands. North is up and east is to the left in all panels. Gray scale is $\sqrt{\log }$ to show faint structure. 


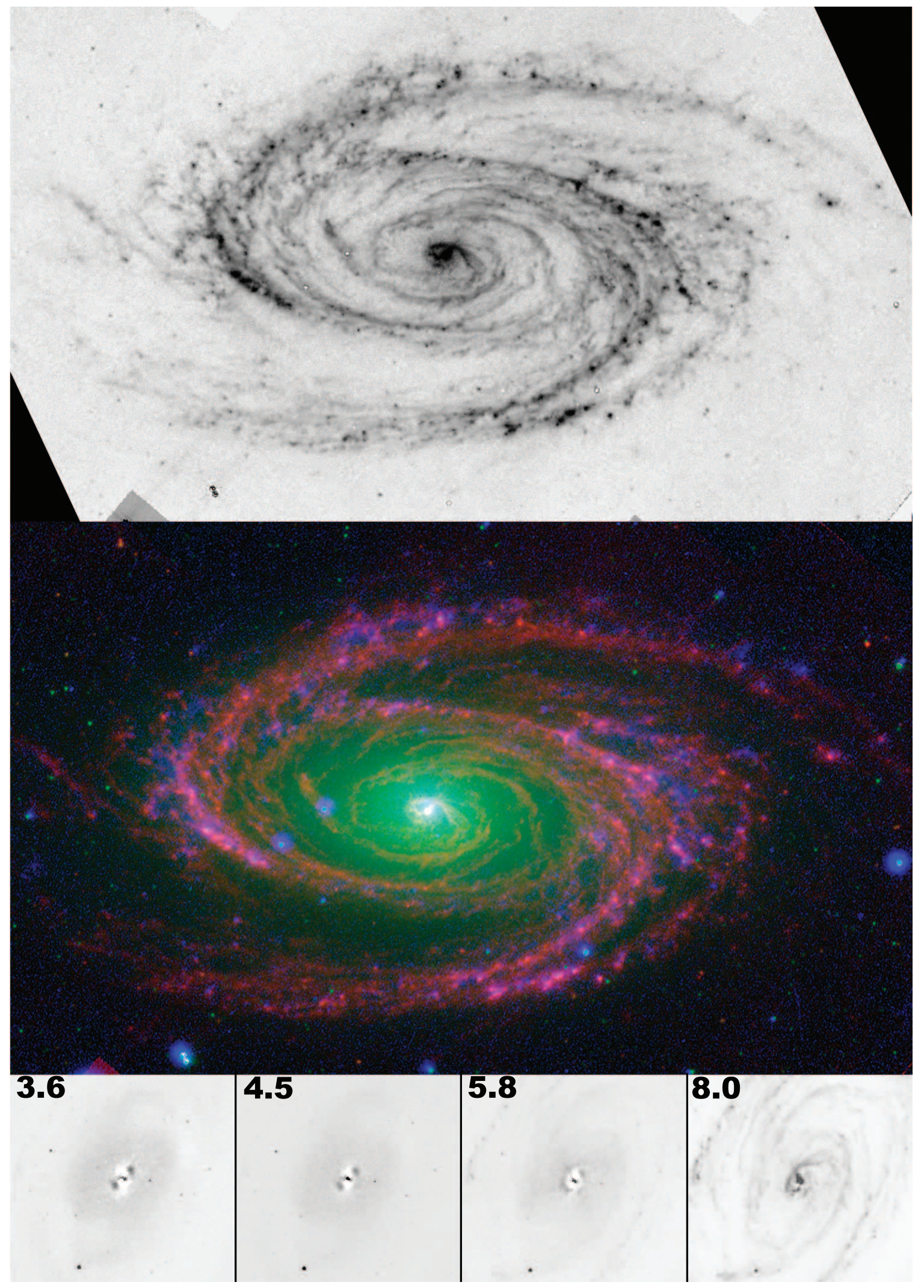

Fig. 3.- Upper panel: $8 \mu \mathrm{m}$ nonstellar radiation. Middle panel: composite color image with blue from the near-ultraviolet (Marcum et al. 2001), green from the $3.6 \mu \mathrm{m}$ image, and red the nonstellar emission from the upper panel. North is $65^{\circ}$ clockwise from the top, and the images are $20^{\prime}$ horizontally. Three regions showing NUV but not $8 \mu \mathrm{m}$ emission are visible in the outer spiral arm above the nucleus. Lower panels: nuclear regions after subtraction of the bulge+disk model. Images are $5^{\prime}=5.3 \mathrm{kpc}$ square, and north is up. Table 1 gives model parameters for $3.6 \mu \mathrm{m}$; values for other bands are similar. White regions near the nucleus represent negative values, i.e., areas where the model surface brightness exceeds the observed surface brightness. 